\title{
INTERVENCIONES FORMATIVAS BASADAS EN WWW PARA GUIAR EL INICIO DE LA PRÁCTICA PROFESIONAL DE LOS DOCENTES
}

\section{María Jesús Gallego Arrufat (*)}

SÍNTESIS: Las tecnologías de la información y la comunicación pueden facilitar el diseño y la realización de buenas intervenciones educativas en el aprendizaje de las tareas de enseñanza del profesorado en su formación inicial, así como a lo largo de su vida profesional. El E-Learning, la CMC (comunicación mediada por computador), los entornos virtuales de comunicación en general, y los foros de discusión asíncrona en particular, ofrecen respuestas basadas en $W W W$ a las necesidades de comunicación de los distintos agentes implicados en las prácticas de enseñanza pertenecientes a escenarios y a estamentos diferentes, aunque con un objetivo común: la guía del inicio de la formación práctica de los futuros profesores.

SÍNTESE: As tecnologias da informação e da comunicação podem facilitar o desenho e a realização de boas intervenções educacionais na aprendizagem das tarefas de ensino do professorado em sua formação inicial, assim como ao longo de sua vida profissional. O E-Learning, a CMC (comunicação mediada por computador), os entornos virtuais de comunicação em geral, e os fóruns de discussão assíncrona em particular, oferecem respostas baseadas em wwW às necessidades de comunicação dos diferentes agentes implicados nas práticas de ensino pertencentes a cenários e a estamentos diferentes, ainda que com um objetivo comum: a guia do inicio da formação prática dos futuros professores.

(*) Profesora titular en la especialidad de Tecnología Educativa, Facultad de Ciencias de la Educación, Universidad de Granada, España. 


\section{PROCESOS DE REFORMA EN LOS PLANES DE FORMACIÓN DE PREGRADO: LAS TIC PARA LA MEJORA DEL PRACTICUM}

El avance de la tecnología y los procesos de cambio e innovación nos conducen progresivamente a un nuevo tipo de sociedad, caracterizada como «sociedad de la información», en la que las condiciones de trabajo, el ocio, las relaciones y la comunicación, o los mecanismos de transmisión de la información, adoptan nuevas formas. Todas estas transformaciones provocan un cambio vital en los individuos. De ahí que su preparación también se esté viendo afectada, necesitando una reformulación en cuanto a contenidos y a formas: sin duda se hace necesario un nuevo planteamiento educativo.

En la mayoría de las instituciones educativas existen procesos de reforma. Los países de la Unión Europea viven en la actualidad políticas de convergencia que afectan a los planes de formación de pregrado en general, siendo prioritarios en el ámbito de la educación superior. Como consecuencia, el diseño institucional de la oferta de formación y de capacitación docente está siendo sometido a un importante proceso de revisión desde el punto de vista de las metodologías y de las estrategias empleadas de enseñanza-aprendizaje. En España, los planes de estudio de educación se dirigen a la búsqueda de estándares para la convergencia. Por citar algunos, los principales proyectos en el marco del Espacio Europeo de Educación Superior (EEES) que se están desarrollando en nuestro contexto son:

- Proyecto Andalucía: proyecto a nivel autonómico que desarrolla la «Experiencia Piloto para la Implantación del Crédito Europeo (ECTS)» en Andalucía, referida a las titulaciones de magisterio. Se creó por iniciativa de la Secretaría General de Universidades e Investigación de la Consejería de Educación y Ciencia de la Junta de Andalucía.

- Proyectos de la Agencia Nacional de Evaluación de la Calidad y Acreditación (ANECA), en el conjunto del Estado español, para la elaboración del mapa de titulaciones «La adecuación de las titulaciones de Maestro al Espacio Europeo de Educación Superior», y «Pedagogía y Educación Social».

En América Latina también encontramos significativos procesos de reforma, teniendo en cuenta, además, la diversidad de institucio- 
nes formadoras existentes para la formación del profesorado (véase Pascual, 1999) y la heterogeneidad dentro de ciertos parámetros comunes como elemento esencial para la reflexión.

En la Universidad de Granada, el grupo de investigación FORCE (Formación Centrada en la Escuela) -al que pertenezco- viene realizando una serie de estudios dirigidos al análisis del practicum en distintas titulaciones universitarias, habiendo enfocado su interés en la formación práctica del profesorado y del pedagogo. Entre ellos destaco los más recientes (algunos en curso de realización):

- Proyecto de investigación «Análisis de practicums de carreras universitarias y descripción de las expectativas de profesores, estudiantes y personal responsable de prácticas 20012003», subvencionado por el Ministerio de Ciencia y Tecnología.

- Investigación «Análisis comparado de la formación práctica en el sistema universitario como base de evaluación y mejora», de la convocatoria Estudios y Análisis. UGR, 2003.

- Investigación «La formación práctica del estudiante universitario y los códigos deontológicos del ejercicio profesional», perteneciente al Programa de Estudios y Análisis (convocatoria de 2001) del Ministerio de Educación, Cultura y Deporte.

No cabe duda de que cualquier proceso de reforma debe descansar en las lecciones aprendidas, derivadas de las respuestas que obtengamos de interrogantes como: ¿por qué cambiar?, ¿estamos preparados para cambiar?, y, tras experimentar y evaluar las innovaciones, ¿qué/cómo hemos cambiado?, ¿qué lecciones hemos obtenido?, ¿qué falta por cambiar?

Las investigaciones citadas han puesto de manifiesto que los objetivos formativos y profesionales en materia de practicum universitario necesitan ser reformulados, estableciendo y adecuando los criterios docentes y de evaluación del alumnado de cada titulación. El análisis de los datos del Cuestionario sobre Enseñanza Práctica Universitaria, FORCE, 2001 (empleado en la investigación citada sobre una amplia muestra de estudiantes de siete titulaciones de la Universidad de Granada), pone de relieve la necesidad de mejorar la vinculación de la formación para la obtención del título con la inmersión profesional que 
debe iniciarse en esta etapa, dando relevancia a la formación práctica, orientación sobre mercado de trabajo y oportunidades profesionales vinculadas a la carrera. La mayoría de los pregraduados advierte en su formación una orientación muy académica, permitiendo escasas posibilidades de aplicar los conocimientos adquiridos (excepto durante el practicum en los centros, y con ciertas limitaciones). En el caso de los futuros docentes ( $N=218$ ) se detecta una importante insatisfacción a la que es preciso prestar atención:

- Un $70 \%$ de los estudiantes de magisterio percibe como escasas las ayudas que han recibido de los profesionales para planificar y llevar a cabo intervenciones en la práctica, habiéndolas tenido que realizar alguna vez (33\%) por propia iniciativa.

- Aprecian que es más frecuente el empleo de equipos tecnológicos para la realización de prácticas simuladas (en laboratorio) que su integración en el desarrollo del plan de prácticas en las escuelas, incluyendo su uso para la observación/descripción de la práctica profesional en la realidad para la evaluación o diagnóstico; y para la intervención profesional o el tratamiento.

- Además, es resultado común a los distintos títulos universitarios de grado, con ligeras variaciones porcentuales, que los estudiantes expresen la necesidad de disponer de foros en los que puedan presentar y discutir su experiencia práctica.

Detectadas estas carencias derivadas de datos como los anteriores, es preciso extender a los estudiantes en prácticas la oferta de servicios proporcionados a través de las tecnologías. Si bien el acceso a la información es un objetivo ya conseguido o que pronto va a serlo, la necesidad y la exigencia de comunicación para que el estudiante cuente con herramientas de uso sencillo que favorezcan la transición de la formación académica a la práctica profesional, es un objetivo prioritario. El requerimiento de foros de discusión comunes hechos a medida es una posibilidad que las TIC deben aportar para la mejora del practicum a través de la orientación, el asesoramiento y la interacción grupal, que facilita el uso de Internet por parte de estos usuarios. El E-Learning viene a ser una fórmula que permite que las herramientas empleadas en los entornos virtuales se conviertan en instrumentos útiles, de cara a la innovación en la formación de pregrado en general, y del profesorado en particular. 
Algunas otras investigaciones en esta línea han detectado la necesidad y/o han sugerido modelos básicos de utilización de nuevas tecnologías en el practicum de las titulaciones de magisterio. Valga citar la investigación dirigida por esta autora, titulada «Uso de los medios en el practicum de magisterio» (Martínez Serrano, 2003), o el «Proyecto Practicum» del grupo de investigación de la Universidad de Málaga sobre experimentación de las nuevas tecnologías, en especial multimedia y redes informáticas (campus virtual), para la mejora de la formación del practicum en las distintas especialidades de enseñantes de los niveles de educación infantil y educación primaria, <http://www.ieev.uma.es/tecedu/ proyec/practic/practi.htm>.

Como ya he indicado, el E-Learning es hoy una respuesta de las TIC para la innovación en la formación de pregrado del profesorado, por ser fórmula que pone en práctica un modelo de formación on-line que se dirige hacia el fomento de la participación del alumno como medio fundamental del aprendizaje, y en el que el profesorado ejerce el rol de guía de conocimientos. Bajo los parámetros del E-Learning, de la formación basada en TIC, de la telemática educativa, existen diferentes y posibles enfoques, desde la educación a distancia tradicional apoyada en el uso de las TIC, a la enseñanza síncrona remota que utiliza los avances tecnológicos para poder llevarse a cabo (ejemplo, cursos a través de videoconferencia), pasando por sistemas tradicionales de educación presencial mejorados por el uso de los medios telemáticos para una mejor intercomunicación entre alumnos y profesores (aprovechando el potencial que Internet ofrece), entre otros. Cada vez es más habitual el desarrollo de experiencias formativas basadas en la conjunción de modalidades presenciales y no presenciales. La mezcla de formación online con formación presencial es una tendencia que en la educación superior se conoce como Hybrid Universities, proliferando en la actualidad innumerables experiencias en esta línea en Europa, en Iberoamérica, y a nivel internacional. Sin duda, en un contexto real la inmersión en la práctica escolar de un futuro docente es un proceso que conlleva siempre una socialización En caso de llegar a su virtualización total, no permitiría degustar, probar, sentir las escuelas, su cultura, siendo la tecnología de comunicación complementaria. Para los agentes la modalidad no presencial en exclusiva no es deseable. Sin embargo, el profesorado y los estudiantes comienzan a solicitar oportunidades de capacitación en entornos virtuales de comunicación complementarios, en los que es posible favorecer, promover y mejorar la intercomunicación entre los implicados en el practicum: alumnos, tutores, supervisores. 
Basándonos en estas premisas, consideramos que es preciso experimentar la eficacia de las intervenciones desde la investigación y el análisis de experiencias formativas destinadas a la mejora de la calidad de la formación práctica del profesorado. Y aunque la base de las prácticas de formación (practicum) reside en el contacto directo con la acción profesional real en los centros escolares, Internet ofrece posibilidades que deben ser valoradas desde la perspectiva de los usuarios como mecanismo de información y comunicación para conseguir una adecuada iniciación en la profesión docente.

\section{EL ASESORAMIENTO AL FUTURO DOCENTE EN ENTORNOS DE COMUNICACIÓN MEDIADA POR ORDENADOR}

La utilización de entornos de comunicación mediada por ordenador en la orientación inicial del aprendizaje práctico requiere plantearse qué competencias son necesarias como punto de partida, $y$, al tiempo, qué competencias van a generarse como resultado (o al menos es propósito de la enseñanza que se consigan como efecto, consecuencia o competencias que el egresado poseerá). Lo fundamental podría establecerse en los términos de los que parte Braslavsky (1999) en su análisis "¿Qué competencias básicas tiene que tener un profesor para poder conducir fértiles procesos de enseñanza-aprendizaje en el siglo XXI?». La respuesta puede llevar al establecimiento de largas listas de cualidades y de conocimientos que el profesorado debe tener. Además, también es cierto que las instituciones educativas necesitan nuevas competencias para adaptarse a la Sociedad de la Información (Cebrián, 2003), porque no podemos hacer recaer todo el peso de la reforma sobre el profesorado, y menos cuando tratamos de repensar la formación de pregrado desde una posición cuyo eje es la innovación tecnológica como base de las metodologías activas centradas en el estudiante.

De todos modos, las directrices para el establecimiento de parámetros desde los que establecer las bases para el diseño de los programas de formación práctica inicial podrían agruparse en competencias básicas en TIC para los docentes, por un lado; y, por otro, competencias genéricas para la profesionalización basadas en el aprendizaje reflexivo. No hacemos en este lugar un análisis de las mismas, pero sí de su concepción: «Toda competencia involucra, al mismo tiempo, conocimientos, modos de hacer, valores y responsabilidades por los resultados de lo hecho» (Ropé y Tanguy, 1994, cit. por Braslasvky, 1999), utilizán- 
dose en el sentido de la capacidad de hacer con la de saber (técne + logos), y con conciencia sobre las consecuencias de ese hacer. En las prácticas de enseñanza, las competencias profesionales comprenden el saber (competencia técnica), saber hacer (competencia metodológica), saber estar (competencia participativa) y saber ser (competencia personal).

En el contexto de las metodologías activas del E-Learning, los procesos de tutoría y asesoramiento son complejos, aunque pueden agruparse en dos modalidades: tutoría electrónica (virtual) y foro de discusión (lista de distribución o comunidad de aprendizaje) ${ }^{1}$. El uso educativo de las TIC incluye múltiples posibilidades, como el empleo de Internet en el aula, o las actividades colaborativas en red basadas en herramientas de trabajo, como CSCL (Computer Support for Cooperative Learning), de las que no nos ocupamos en este trabajo. Del mismo modo, la comunicación simultánea y en tiempo real a través de chat, videoconferencia, pizarra compartida y otros sistemas interactivos de comunicación síncrona de indudable importancia en entornos de comunicación mediada por ordenador, tienen entidad en sí mismos, dando lugar al aula virtual (entre las múltiples posibilidades que tampoco analizamos ahora).

Con la pretensión de establecer y delimitar el concepto de «foro de discusión» en el presente estudio, partimos de que, en general, los posibles foros a través de redes de ordenadores se definen como «un espacio para el intercambio de ideas, al mismo tiempo que una fuente de información sobre temas específicos». Los foros se organizan en temas. Cualquier usuario puede abrir un nuevo tema de conversación, al que se añaden los sucesivos mensajes sugeridos por o en respuesta al inicial. Permitiendo y favoreciendo la colaboración entre docentes y estudiantes más allá de los límites físicos y académicos del centro educativo al que pertenecen, facilitan que grupos de alumnos y de profesores constituyan comunidades virtuales de colaboración en determinados temas o campos de estudio. Las comunidades virtuales son definidas como espacios de intercambio comunicativo entre colectivos con un interés común. Las modalidades comunicativas que se establecen en un foro permiten el desarrollo de espacios de relación social que

${ }^{1}$ Indudablemente, un examen conceptual Ilevaría a establecer distinciones entre ellos, pero en este trabajo quedan englobados funcionalmente como foros de discusión diversas modalidades asíncronas de comunicación, en las que destacan el intercambio y la comunicación entre profesionales y personas con intereses comunes. 
promueven el aprendizaje interactivo. Por ello, pensamos ante todo que el empleo que Internet ofrece puede mejorar la intercomunicación entre alumnos, profesores tutores de centros de prácticas y profesores universitarios, siendo general la utilidad y el beneficio pedagógico obtenidos.

Además, el saber acumulado en la tradición didáctica ha puesto de manifiesto que, tanto la tutoría individual como el asesoramiento al grupo, son dos modalidades esenciales en la enseñanza presencial, al tiempo que se aprecian en los sistemas educativos como aquellas que han demostrado ser imprescindibles en esta forma de enseñanza. Por lo que respecta al aprendizaje, las necesidades y los estilos de estudio de cada estudiante conllevan la adaptación y la diversificación de estrategias adecuadas a las diferencias individuales que presentan, estableciéndose procedimientos metodológicos complementarios según modos de construcción del aprendizaje (actitudes, capacidades, estilos cognitivos, ritmos de trabajo, etc.), referido en este caso al práctico de las tareas de enseñanza.

Contar con un espacio compartido en la red accesible desde cualquier máquina que disponga de conexión a Internet, posibilita complementar la comunicación cara a cara destinada a la enseñanzaaprendizaje, ya sea del individuo o del grupo. El valor añadido radica en la interactividad, concepto inherente a los procesos de comunicación, que permite que emisor y receptor intercambien continuamente sus papeles. Así, todos los participantes en el foro de discusión pueden actuar como consejeros, al intervenir sobre los temas a medida que van surgiendo, desarrollándose los procesos grupales mediante tutoría entre iguales, aprendizaje cooperativo y/o colaboración.

El asesoramiento al futuro docente se establece desde ideas metodológicas en las que el tutor asume los papeles de facilitador, moderador del discurso, orientador y guía, que ya se perfilaban como los más adecuados al emplear medios informáticos, aun sin las plataformas y las conexiones actuales (Gallego, 1995). Se enfatiza su función en las tutorías, al no ser ya protagonista del proceso, pues ahora lo es el estudiante. El tutor es un moderador que adopta el rol de asistente que sólo ayuda al alumno cuando éste realmente lo requiere, mientras que el alumno asume un papel autorregulativo y autointerrogativo. De ahí que algunos tutores (e incluso mentores) aún no iniciados en la transformación y adaptación de sus roles docentes ante las TIC pueden creer que sus tareas disminuyen debido a la automatización de procesos, o, incluso, sentirse desplazados por la interacción estudiante-máquina como ele- 
mento motivador para los estudiantes, pero que juzgan poco adecuado para una intervención formativa en la que siempre será imprescindible la transmisión de información por su parte. Muy al contrario, la investigación sobre la innovación didáctica y sobre las nuevas funciones que las TIC incorporan ha demostrado que se trata de falsas expectativas (o de creencias no adaptativas), y que lo cierto es que la dedicación a trabajos relacionados con el asesoramiento en entornos de comunicación mediada por ordenador aumenta mucho las responsabilidades docentes y las horas que se dedican a ello.

Las funciones del tutor, así como las estrategias que debe desarrollar para el asesoramiento de los estudiantes en prácticas en un entorno virtual, serán aquellas que ayuden a construir y a impulsar un modelo de supervisión flexible, donde prime la actividad y la construcción del conocimiento práctico por parte del estudiante, superando las limitaciones temporales y las distancias geográficas. Deberá desarrollar sobre todo funciones tecnológico-comunicativas, ser usuario experimentado de las herramientas telemáticas -al tiempo que soporte de los alumnos para el acceso y la recuperación de la información-, e interaccionar en entornos diferentes a los de formación presencial cara a cara; y también funciones pedagógico-didácticas en la medida en que organice, diseñe y evalúe la situación mediada de aprendizaje que se lleva a cabo en el foro de discusión, adaptando sus estrategias de modo dinámico y flexible durante el transcurso del mismo.

En esencia, las características pedagógicas esenciales en el entorno virtual (comunicación interpersonal, seguimiento de las intervenciones del alumno, etc.) son la base desde la que se puede realizar el asesoramiento en la formación práctica de los futuros docentes que se incorporan por primera vez al mundo escolar, con objeto de conseguir analizar y valorar las experiencias vividas en el ámbito escolar; identificar problemas pedagógicos y hallar posibles formas de solución; recapacitar sobre la naturaleza de la educación en función de las experiencias vividas; lograr reflexiones y análisis más amplios acerca de las relaciones escuela-sociedad, sobre el funcionamiento del sistema educativo, o, en definitiva, acerca de los dilemas percibidos en la relación teoríapráctica.

Las dudas, los problemas, las preguntas, las revisiones de los estudiantes en prácticas, si estos lo desean, son compartidas con los tutores del centro y de la universidad, y con compañeros de otros centros en similar situación, apreciando una importante ventaja en el foro de 
discusión al encontrar respuestas, comentarios y contrastes cuya rapidez dependerá de la asiduidad de acceso de cada uno, siendo recomendable la consulta diaria. En este sentido, es conveniente distinguir el mensaje del foro (que se lee/responde a diario) integrado en el debate compartido, por una parte, y, por otra, el conjunto de reflexiones y de vivencias que se reflejan en el diario de prácticas completo, que debe formar parte del itinerario personalizado de cada uno.

\section{DISEÑO DEL MODELO PEDAGÓGICO}

El modelo de apoyo a la docencia que es posible llevar a cabo en la mayoría de las universidades contempla intervenciones formativas presenciales y virtuales, existiendo además otros modelos de universidades abiertas, de campus virtuales, etc., que, en clara expansión, sustentan cierta internacionalización del conocimiento y de la formación. Como ya indiqué, las intervenciones formativas pertenecientes a la etapa de formación básica y de socialización profesional se apoyan en un modelo híbrido de intervención que combina actividades presenciales y virtuales, que son establecidas desde el plan oficial de prácticas existente en la institución. A grandes rasgos, el practicum está dividido en dos tramos: el primero general, de observación y toma de contacto con la realidad escolar, y el segundo más especializado, durante el que el estudiante en prácticas pasa de la observación a la intervención guiada. Existen distintos sistemas y procedimientos institucionales, aunque por lo común se realizan al final de los estudios de formación de profesorado (o al menos en el último año), y su duración comprende la mitad del mismo con dedicación de jornada completa. En el practicum, las principales actividades presenciales de asesoramiento incluyen diversas reuniones entre los profesores universitarios tutores y los futuros docentes en prácticas, antes, durante y después de visitar la escuela, que, a su vez, se corresponden con el seminario inicial, los seminarios intermedios y el seminario final.

Los seminarios intermedios realizados por los supervisores (profesores universitarios tutores de prácticas de los estudiantes) en el plan de prácticas figuran como reuniones presenciales con el grupo de alumnos que tengan asignado, una tarde a la semana en tanto permanezcan en las escuelas, estancia que se corresponde con el horario lectivo normal del centro al que acuden (todas las mañanas, y, en muy escasas ocasiones, una o dos tardes). De este modo, se pretende que la realización 
de las prácticas en un escenario real de clases de educación infantil y de educación primaria tenga un seguimiento en la universidad. El seminario de supervisión se convierte así en una estrategia destinada al apoyo y guía del estudiante en el inicio de su práctica profesional de cara a la obtención del máximo provecho de su estancia en el centro, donde se aplican y ponen a prueba las concepciones por las que se orienta la propia acción docente. En la literatura sobre el profesorado debutante es ya clásica la descripción de esta etapa como choque con la práctica, estando Ilena de tensiones y conflictos, y con los sentimientos resultantes de desconcierto, angustia, inseguridad e inestabilidad. El/la futuro/ a profesor/a comienza a componer nociones de una determinada cultura profesional, cuyas pautas se empiezan a asumir y a consolidar a partir de estas primeras experiencias de práctica educativa, gracias a múltiples procesos de aprendizaje generados en el propio centro, en el que colabora activamente con el maestro/a tutor/a. En el modelo pedagógico adoptado existe, además de los referidos, un seminario virtual, que es un foro de discusión abierto en el que se puede explicitar, debatir o compartir experiencias, vinculado de modo asíncrono con este importante período de formación del futuro docente.

\subsection{ESTRATEGIAS BASADAS ENWWW: SEMINARIO VIRTUAL}

Junto con la disponibilidad tecnológica (para que sea posible la participación y el seguimiento en el foro), y la necesidad de comunicación (intereses o motivaciones de los alumnos para intervenir), en un seminario virtual es esencial considerar el contexto de la comunicación para establecer los mecanismos de adecuación al nuevo contexto de asesoramiento.

Las habilidades para la participación en el seminario virtual están referidas no tanto a los aspectos técnicos como a los comunicativos. Respecto a los primeros, la mejor plataforma será la más sencilla y accesible para todos, sin comandos complicados, aunque con los privilegios mínimos para el moderador (rol desempeñado por el profesorado supervisor) y gratuita pero sin excesiva publicidad, como sucede con algunos generadores de sitios web en línea gratis que permiten crear foros, siendo preferible la existente en el servidor institucional al que pertenecen los miembros del grupo. Respecto a los segundos, el entorno virtual requiere ciertas destrezas para la participación como punto de partida para la comunicación, entre las que destacamos la comprensión y la discriminación de los mensajes a leer; distinguir los mensajes que 
deben dirigirse al grupo de los individuales, que se derivarán a la tutoría electrónica y/o presencial; respetar la estructura ramificada del foro enlazando los temas abiertos, empleando para ello los asuntos (subject) aunque no exista una proximidad temporal entre los mensajes, y referenciando al autor del mensaje o de la intervención; discernir si los mensajes son útiles y/o adecuados para el grupo, así como para el contenido concreto objeto del foro; y, en general, cuidar la ambigüedad - la pérdida de información propia de la comunicación mediada por ordenador, sobre todo en la comunicación escrita, reseñando con cuidado los referentes contextuales para que la percepción del entorno sea compartida por el grupo.

El seminario virtual, a través de la modalidad de foro de discusión, se basa en procesos grupales que promueven el desarrollo cognitivo y el aprendizaje entre los alumnos. Para estructurar las interacciones grupales son clásicas las investigaciones de Web y colaboradores (cit. Gallego, 1995), en las que se indican algunos aspectos aplicables a este entorno, con ciertos matices: la especialización de roles, el cuestionamiento recíproco, las explicaciones puntuales, y la búsqueda de controversias versus concurrencias.

En este modelo las principales estrategias son: contrato de prácticas al inicio, en forma de reglas de juego como punto de partida en el que se informa como si se tratase de un tablón de docencia de cuestiones técnicas y organizativas (documentos-guía, actividades a realizar, cómo y cuándo, cómo se llevará a cabo la discusión, el tiempo a invertir y los criterios de evaluación); comunicación de uno al grupo, en forma de sitio donde plantear preguntas y compartir reflexiones con el tutor y los compañeros; escritura libre de reflexiones individuales en forma de lluvia de ideas, tras una actividad realizada en el aula del centro de prácticas; estrategias centradas en el pensamiento crítico (pros y contras, aspectos positivos y negativos, votaciones, categorizaciones); mentorización entre iguales, donde las intervenciones asociadas tras el inicio de un tema se basan en que los alumnos intervienen desempeñando el rol de orientadores y guías de otros colegas que no han encontrado solución a la cuestión planteada, revisadas tanto por el moderador como por el grupo; informe de consenso, animando a contrastar puntos de vista para llegar a conclusiones, favoreciendo la síntesis para sugerir propuestas alternativas de actuación.

Como sucede en el seminario de supervisión presencial, el análisis de casos es la principal estrategia en la que se basan las 
intervenciones formativas de asesoramiento durante las prácticas de enseñanza, que, llevada a un entorno virtual, sigue considerándose fundamental y empleándose para al diagnóstico y la solución de problemas prácticos, desde la reflexión y la comprensión de las posiciones, actitudes y metodologías de los agentes educativos ante los mismos.

La figura representa el modelo de seminario virtual que hemos empleado, aunque siempre es preferible que en cada intervención formativa específica basada en WWW se disponga un modelo ad-hoc centrado en el grupo o comunidad de aprendizaje del que se trate. Los modelos deben ser flexibles, basados en la difusión de creencias y de experiencias personales de los miembros, y, para que el foro de discusión sea una herramienta útil para el moderador-tutor, también deberá abstenerse de intervenir en ocasiones. La excesiva regulación a través de modelos pedagógicos rígidos tampoco sería deseable.

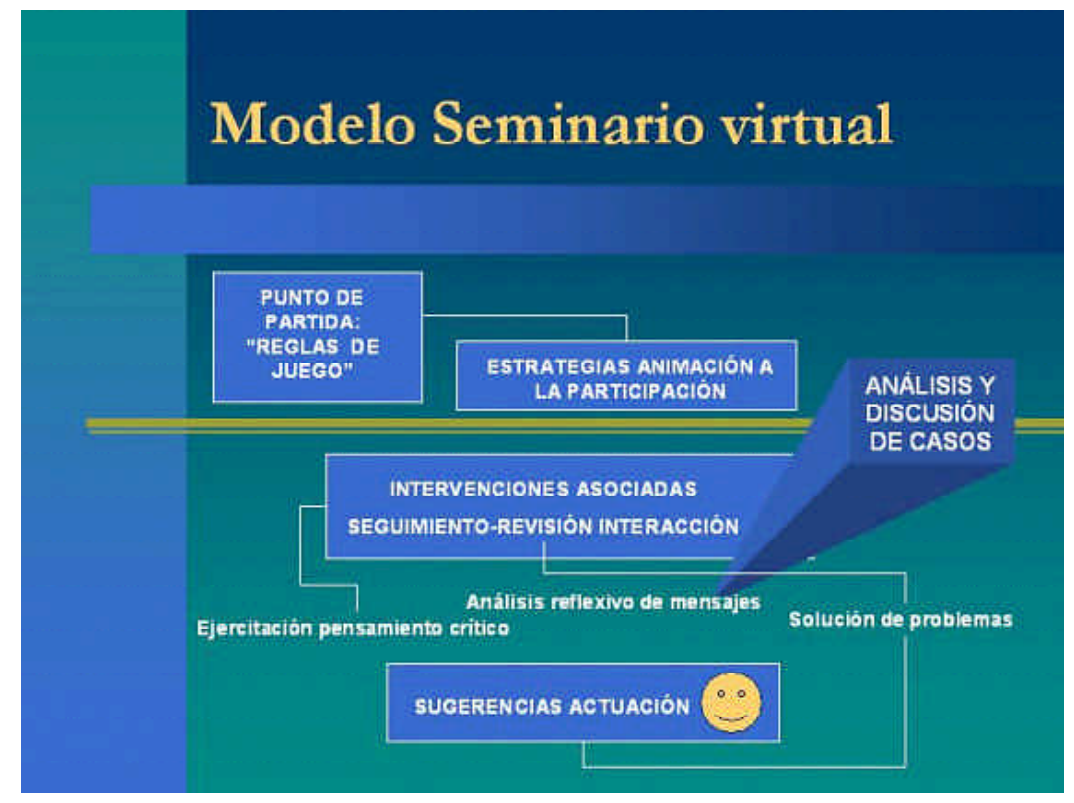

3.2 PRINCIPIOS QUE DEBEN REGIR LA COMUNICACIÓN INTERPERSONAL EN EL FORO DE DISCUSIÓN

- Al principio, importancia del establecimiento de unas reglas de juego muy definidas, referidas tanto a la univocidad y existencia de un lenguaje común como a metas específicas, 
fomentando en todo momento la ayuda mutua entre los miembros del foro como entidad virtual.

- Continuidad y frecuencia en las conexiones, asiduidad diaria de accesos, agilidad y dinamismo (casi sincronía) en las intervenciones del moderador.

- Libertad de expresión, respeto y tolerancia ante las actitudes y las opiniones, rigurosidad en la expresión de conceptos y de conocimientos, orientación y guía especifica del moderador para el análisis y la reflexión.

- Al final, importancia de la obtención de conclusiones y de sugerencias de actuación derivadas de la reflexión sobre las propias intervenciones y las de los colegas.

En su aplicación práctica, es preciso tener en cuenta algunos errores que hay que evitar y los esfuerzos que se deben realizar, entre los que destacamos los siguientes:

- Obviamente, cualquier interacción aparece en forma de mensaje verbal escrito ( $\sin$ mensajes no verbales o paraverbales que ayuden a su comprensión). A menudo quisiéramos intervenciones más precisas y análogas a las realizadas en el aula, porque dominamos más la comunicación presencial. Léase con humor el siguiente fragmento procedente de un chat, como ejemplo de situación que se debe evitar:

«Alumno: ¿Cómo puedo quitar mi PC del escritorio?

Profesor: [confundido] ¿qué pasa, está atornillado a la mesa o qué?

Alumno: noooo, bueno, no sé, ¿qué hago?

Profesor: [más confundido] no... a ver, ¿dónde quieres poner tu PC?

Alumno: En ningún sitio.

Profesor: ¿?

(Pausa, tiempo de espera)

Alumno: profe, quiero QUI-TAR-LO >-I

Profesor: ok. Vamos por partes, ¿eh? Tómate tu tiempo para explicarme lo que quieres hacer y no puedes con más detalle. 
Alumno: He quitado Internet, he quitado el outlook y he quitado "m is docum entos", pero no m e deja quitar "m iPC", y la "papelera de reciclaje" tampoco".

Situaciones cómicas como ésta (o no tanto, porque el alumno llega a escribir en mayúsculas lo que en la red equivale a gritar) también se pueden producir en la comunicación asíncrona, al no ser frecuente el empleo de comillas, signos de puntuación, y, en general, las correcciones ortográficas, semánticas y sintácticas en los mensajes. En un foro hay que cuidar el mensaje enviado; se debe estructurar y redactar con exactitud la intervención antes de hacerlo. Debe ser breve y conciso.

- En la comunidad de usuarios, estudiantes y tutores deben hacer un esfuerzo: el alumno, por respetar las normas ortográficas, de redacción y estilo para la correcta comprensión de su expresión por todos, mientras que el profesor debe conocer y emplear los smileys (o emoticones) más usados por sus estudiantes (ejs. : «-? dubitativo»; «:-/ mosqueado», «:-| serio», «:') emocionado»...).

- Es conveniente la participación de todos, lo cual, en situaciones de comunicación no presenciales, es tan difícil de lograr como en las presenciales. Alguna estrategia de provocación o incitación -obligatoria- puede ser útil para tratar de evitar el lurking (fisgoneo, mironeo), lo que sucede en la mayoría de las listas de distribución (se leen y se consultan los mensajes de otros, pero es menos frecuente enviar los propios). Incluso sería oportuna la distribución de roles análogos a diferentes destrezas sociales para el funcionamiento eficaz del foro, en orden a conseguir el objetivo común de inmersión en todo lo que implica la profesionalización docente, como, por ejemplo, el incitador o provocador, el comandante de preguntas, el monitor del calendario, etcétera.

- Es útil que el supervisor-administrador del foro elabore un módulo dedicado a preguntas frecuentes (FAQ), lo que evitará la mezcla de mensajes anudados sobre temas técnico-organizativos que podrían resolverse consultando un tablón. 
- Son necesarios estudios en los que se analice por qué funcionan los foros de discusión en Internet (Guzdial y Turns, 2000; Lipponen et al. 2003; Dwyer, 2003) para obtener resultados extensibles sobre el desarrollo correcto y efectivo de esta modalidad de comunicación, porque no sólo importa el hecho de que se ofrezcan o dejen de ofrecerse oportunidades de capacitación docente, sino, sobre todo, el resultado obtenido. No es insólito encontrar foros que aprovechan muy poco sus posibilidades, con escasos mensajes, en los que apenas aparecen una o dos intervenciones de estudiantes dirigidas exclusivamente a obtener información sobre la fecha de entrega de los trabajos o sobre el lugar en el que se puede obtener un documento para fotocopiarlo.

Resultados y discusión. Después de observar el funcionamiento del foro de discusión sobre aprendizaje práctico docente durante el transcurso de un tramo de prácticas completo, de realizar una encuesta a un pequeño grupo de usuarios, y de analizar los diarios y memorias de prácticas del conjunto de los futuros enseñantes participantes en el seminario virtual, los resultados que aquí presentamos abordan la naturaleza de la comunicación en red, los temas sobre prácticas de enseñanza que han sido objeto de reflexión, y el valor que los usuarios otorgan a este foro:

- Comunicaciones en la red: Sobre un total de 27 estudiantes, la participación en el foro osciló entre la alta y la media, si tenemos en cuenta el número total de conexiones y la frecuencia de las mismas. Todos leyeron los mensajes del foro con diferente asiduidad, desde a diario hasta una vez a la semana. El número total de mensajes ascendió a 93, y el de participantes con intervención activa representó un 42\% del grupo.

- Principales módulos temáticos en la Network asíncrona: aparecieron cuatro temas de especial relevancia y entidad: disciplina, relaciones con los padres, motivación, religión y escuela. Los tres primeros eminentemente prácticos, y el último algo más teórico-filosófico. Existieron también mensajes híbridos, que podrían encuadrarse en diferentes categorías, como aquellos en los que se expresaron opiniones sobre el dilema de la atención a la diversidad (necesidad de atención individualizada permanente versus integración 
social). Las reflexiones, opiniones, argumentos, razonamientos, cuestionamientos, dudas y sugerencias también se refirieron a otros temas, entre los que destacaron: gestión del aula, problemas de aprendizaje, problemas de conducta, dificultad para enseñar a la clase completa, a grupos y a individuos, etcétera.

- Valor que los estudiantes otorgaron a esta modalidad de seminario: como en cualquier intervención formativa, hubo quienes opinaron que había funcionado bien, a pesar de la dificultad que supuso la disponibilidad, y en su memoria de prácticas advirtieron: "Incluyo un comentario sobre el seminario virtual, agrupando los mensajes por temas, y también los mensajes. Personalmente me ha servido mucho, pero tengo que reconocer que ha sido gracias a un amigo que me los ha ido imprimiendo, porque yo no tenía modo de consultarlo» (019). Y aquellos para quienes no había funcionado: "Yo, esto del foro, la verdad es que no lo he utilizado mucho, porque ni tengo ordenador ni soy amante de la informática; además, por las tardes tengo clases y tampoco tengo tiempo para conectarme»(002).

Algunas otras opiniones sobre el modelo de aprendizaje práctico basado en WWW que expresaron los estudiantes en sus memorias de prácticas fueron:

- Declaraciones de carácter general: «Creo que está bien escuchar los problemas, dudas y opiniones de los demás; nos ayudan de algún modo a abrir la mente y a ver las cosas de otro color. Siempre pensamos que nuestros problemas son los peores o que somos los únicos con problemas. En mi opinión, el seminario virtual (foro) nos ha ofrecido muchas ventajas y una mayor facilidad a la hora de comunicarnos y de resolver dudas»(001).

- Percepciones y expectativas del estudiante: «Principalmente, en el foro he buscado temas que se identificasen con los problemas que yo iba teniendo» (022).

- Visión sobre flexibilidad y dinamismo del entorno virtual: "Como ya se va acercando la fecha de entregar el cuaderno de prácticas y van surgiendo más dudas, el foro se pone más interesante; unos y otros nos respondemos, nos atrevemos a 
contar anécdotas y a preguntar [...]. El día en que finalizó el seminario nuestra supervisora se despidió de nosotros con un agradable mensaje. La verdad es que a todos los que compusimos el foro nos dio pena que se terminara [...]. En el foro la gente ha seguido hablando; algunos nos hemos seguido comunicando, aunque otros se han despedido» (003).

- Conclusión sobre la eficacia del modelo: «Ha sido muy gratificante hacer frente a problemas de atención y motivación, dando ideas a mi tutora que adopté del foro del practicum. Al principio no veía muy claro lo del sistema de recompensas, pero cuando $F$. dijo que a él le había funcionado fue cuando hablé con ella. Intenté motivarlos con caramelos, pero sólo a aquellos que se portaran bien, diciéndoles también lo importante que es escribir y leer para hacerse mayores»(012).

\section{CONCLUSIONES Y SUGERENCIAS}

El inicio de la práctica profesional apoyada en un grupo de trabajo que se comunica mediante un foro de discusión, además de los recursos informativos que ofrece, anima a sus miembros a compartir experiencias y a colaborar ayudando a establecer buenas prácticas. Sin duda, sería deseable la extensión y difusión de investigaciones para averiguar el éxito de los seminarios virtuales con futuros docentes de múltiples instituciones de formación de pregrado, y, en cualquier caso, el diseño a medida de los mismos, invitando al profesorado experimentado a participar en ellos. También sería necesario un análisis particularizado del contenido de los mensajes, en orden a averiguar sobre qué tipo de competencias básicas reflexionan los estudiantes en prácticas para detectar sus necesidades, y de éstas derivar líneas para la reforma de los planes de estudio de sus titulaciones.

Orientaciones para el diseño de intervenciones formativas en entornos virtuales:

- La disponibilidad y el acceso a las herramientas telemáticas es condición necesaria, aunque no suficiente, para el desa- 
rrollo de las modalidades basadas en www de tutoría y asesoramiento en la formación práctica inicial.

- Es preferible la comunicación asíncrona, dada la necesidad de adecuar ritmo y horarios de trabajo a los diferentes usuarios.

- Se debe promover la participación de los tutores de los centros de prácticas, desarrollando estrategias específicas que fomenten las interrelaciones con colegas con necesidades e intereses próximos a su propio perfil y especialidad, estimulando su intervención mediante incentivos y reconocimientos profesionales por parte de la administración educativa.

- En cualquier caso, deben existir espacios de relación social entre los participantes: seminarios presenciales, reuniones periódicas, encuentros y jornadas de intercambio y reflexión, desde los que se apoye y fomente la colaboración establecida en el entorno virtual.

- Las intervenciones formativas colectivas deben tender a alcanzar un equilibrio entre la cooperación y la colaboración y la autonomía y la individualización, atendiendo de modo complementario a las necesidades particulares desde el establecimiento de canales de comunicación privativos (bidireccionales), fundamentales también para el inicio de la práctica profesional de los futuros docentes: supervisortutor, supervisor-estudiante, tutor-estudiante.

En última instancia, la mejora del funcionamiento de este modelo descansa, desde el punto de vista práctico, en la necesidad de que se disminuya la dimensión de los trabajos que se entregan como memorias de prácticas, y se dedique más tiempo a la interaccióncomunicación entre profesor-alumno en prácticas, profesor tutor en el centro y profesor supervisor en la facultad, tal como se ha venido sugiriendo en los sim posios intemacionales sobre el practicum celebrados en Poio, España (ver, p. ej. Pavón, 1998).

Como conclusión, no puedo dejar de reconocer que la participación del profesorado tutor en los centros de prácticas en foros de discusión como el descrito es una posibilidad difícilmente realizable, teniendo en cuenta que la relación institucional entre centros formativos 
y centros de prácticas pasa por ser una relación de carácter turbulento, conflictivo, e, incluso, disonante. A través de la realización de experiencias en las que su participación voluntaria se desarrolle mediante fórmulas descritas en otro lugar (Gallego, 2001), podrían obtenerse lecciones de la práctica desde las que reelaborar modelos de colaboración para avanzar y mejorar las relaciones interinstitucionales universidad-escuela. Los seminarios virtuales no deben alejar al practicum de su verdadera función de acercamiento de la formación inicial al mundo real del ejercicio de la profesión en condiciones normales -difíciles en la mayoría de ocasiones- y no en condiciones de laboratorio, y menos aún en entornos virtuales, como bien pueden opinar los docentes experimentados con actitudes poco favorables al uso de las TIC.

\section{BIBLIOGRAFÍA}

BRASLAVSKY, Cecilia (1999): «Bases, orientaciones y criterios para el diseño de programas de formación de profesores», en Revista Iberoamericana de Educación, núm. 19, pp. 9-11, Madrid, OEI.

CEBRIÁN, Manuel (2003): «Análisis, prospectiva y descripción de las nuevas competencias que necesitan las instituciones educativas y los profesores para adaptarse a la sociedad de la información", en Revista de Medios y Educación, 20, pp. 73-80 Pixel-Bit.

DWYER, Vincent (2003): Por qué funciona ClinMicroNet: estudio de un foro de discusión en Internet como red de conocimiento. Trabajo de Doctorado TD03-006, 2003, $<$ http://www.uoc.edu/in3/esp/index.htm\#>.

GaLlego, M. Jesús (1995): «Análisis de la acción docente en el aula de informática: implicaciones para una didáctica de la informática», en Qurrículum, 10-11, pp. 77-102.

- (2001): «El profesorado y la integración curricular de las nuevas tecnologías», en Manuel Area (coord.): Educar en la Sociedad de la Información, Bilbao, Desclée de Brouwer, pp. 283-497.

GUZDIAL, Mark y TURNS, Jennifer (2000): «Effective Discussion Through a Computer-Mediated Anchored Forum», en Journal of the Learning Sciences, 9 (4), pp. 437469.

LIPPONEN, Lasse et al. (2003): «Patterns of Participation and Discourse in Elementary Students: Computer-Supported Collaborative Learning», en Learning and Instruction, 13 (5), pp. 487-509.

MARTínEZ, M. Carmen (2003): Uso de los medios en el prácticum de magisterio, Tesis Doctoral inédita, Universidad de Granada. 
OCKER, Rosalie J. (2002): «Exploring the Mediating Effect of Group Development on Satisfaction in Virtual and Mixed-Mode Environments», en e-Service Journal, 1 (3), Summer.

PASCUAL, Enrique K. (1999); «Informe de la formación de profesores de pre y postgrado desde las instituciones formadoras más importantes en América Latina», en Revista Iberoamericana de Educación, núm. 19, pp. 229-269, Madrid, OEI. 


\title{
Contactar
}

Revista lberoamericana de Educación

\author{
Principal OEI
}

